\title{
IBS and Expected Luminosity Performance For RHIC Beams At Top Energy With 56 MHz SRF Cavity
}

Alexei Fedotov

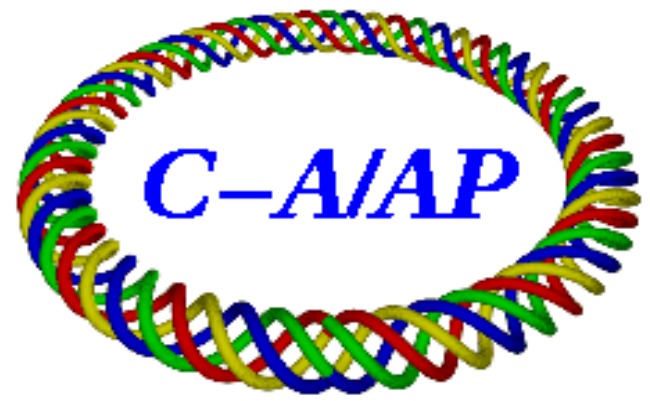

\section{Collider-Accelerator Department Brookhaven National Laboratory Upton, NY 11973}

Notice: This document has been authorized by employees of Brookhaven Science Associates, LLC under Contract No. DE-AC02-98CH10886 with the U.S. Department of Energy. The United States Government retains a nonexclusive, paid-up, irrevocable, world-wide license to publish or reproduce the published form of this document, or allow others to do so, for United States Government purposes. 


\title{
IBS AND EXPECTED LUMINOSITY PERFORMANCE FOR RHIC BEAMS AT TOP ENERGY WITH 56 MHZ SRF CAVITY
}

\author{
Alexei Fedotov \\ C-AD, Brookhaven National Laboratory
}

\section{Introduction}

The purpose of RF system in RHIC is to capture injected bunches, accelerate them to the top energy, and store bunches at the top energy for many hours. The accelerating RF system operates at harmonic number $h=360$ of the particle revolution frequency $f=78.196 \mathrm{kHz}$, which corresponds to 28.15MHz. The storage RF system accepts the shortened bunches at top energy and provides longitudinal focusing to keep these bunches short during the store time (collision mode). The storage system operates at harmonic number $h=7 \times 360=2520$, which corresponds to an RF frequency of $197.05 \mathrm{MHz}$ [1].

Recently, an upgrade of storage RF system with a superconducting $56 \mathrm{MHz}$ cavity was proposed [2]. This upgrade will provide significant increase in the acceptance of storage RF bucket. Presently, the short bunch length for collisions is obtained via RF gymnastics with bunch rotation (called "re-bucketing), because the length of $197 \mathrm{MHz}$ bucket of $5 \mathrm{nsec}$ is too short to accommodate long bunches otherwise. However, due to bucket non-linearity and hardware complications some increase in the longitudinal emittance occurs during re-bucketing. The $56 \mathrm{MHz}$ cavity will produce sufficiently short bunches which would allow one to operate without re-bucketing procedure.

This Note summarizes simulation of beam evolution due to Intra-beam scattering (IBS) for beam parameters expected with the $56 \mathrm{MHz}$ SRF cavity upgrade. Expected luminosity improvement is shown both for $\mathrm{Au}$ ions at $100 \mathrm{GeV} /$ nucleon and for protons at $250 \mathrm{GeV}$.

\section{Performance of Au ions beams at top energy of $100 \mathrm{GeV} /$ nucleon}

IBS limits the present performance of the RHIC collider with heavy ions. To achieve required luminosities for a future upgrade of the RHIC complex, the Collider-Accelerator department at BNL has been developing several approaches to counteract IBS such as electron and stochastic cooling [2, 3]. Stochastic cooling in the longitudinal direction was already successfully implemented in RHIC, suppressing longitudinal emittance growth due to IBS in one of RHIC rings. Implementation of stochastic cooling in the transverse plane is presently under development [3].

In addition, the work is being done on the reduction of the transverse IBS growth rate with a modification of the RHIC lattice. This approach is less expensive and intrusive than cooling. As a first step in this direction, the new RHIC lattice was developed over several dedicated Accelerator Physics Experiments (APEX) [4, 5]. In this Note, IBS for RHIC beams is simulated with the new "dAu82" lattice, used in 2008 RHIC physics run in Yellow ring. This newly implemented lattice has $95^{\circ}$ horizontal phase advance per arc cell and a transition gamma of $\gamma_{\mathrm{t}}=26.6$. 
At injection energy typical 95\% longitudinal emittance of $\mathrm{Au}$ ions is $\mathrm{S}_{95 \%}=0.3-0.5 \mathrm{eV}-\mathrm{s} / \mathrm{nucleon}$. The emittance is growing during the ramp reaching the values of about $0.8 \mathrm{eV}$-s/nucleon at the top energy, which corresponds to a full bunch length of $9.7 \mathrm{nsec}$ with $28 \mathrm{MHz}$ RF. Such bunch length is too long to fit into 5.1 nsec bucket length of $197 \mathrm{MHz}$ RF. The short bunch length for collisions is thus obtained via RF gymnastics with bunch rotation (called "re-bucketing"). However, some increase in the longitudinal emittance occurs during this procedure. Longitudinal emittance after rebucketing is about $1.5 \mathrm{eV}$-s/nucleon. Also, as a result of re-bucketing, significant intensity spill into the neighbouring buckets occurs as well. Typically, about $30 \%$ of bunch intensity is spilled into the neighbouring buckets. This would correspond to a two-fold reduction in useful luminosity if these satellite buckets would not contribute to the collisions within the detector vertex. Of course, bunches in the satellite buckets still collide within the vertex, making luminosity reduction caused by the spill slightly less severe.

The RF upgrade with 56MHz cavity will produce sufficiently short bunches which would allow one to operate without re-bucketing procedure. This would prevent longitudinal emittance increase as a result of re-bucketing and eliminate intensity spill in the neighbouring buckets thus maximizing useful luminosity. Comparison of RF parameters for present 28 and $197 \mathrm{MHz}$ RF and planned 56 $\mathrm{MHz} \mathrm{RF}$ is given in Table 1 . Also note that for longitudinal emittance of $0.8 \mathrm{eV}$-s/nucleon the full bunch length with $56 \mathrm{MHz}$ RF is only $4.8 \mathrm{nsec}$, which is smaller than the length of $197 \mathrm{MHz} \mathrm{RF}$ bucket. This suggests that further shortening of bunch length may be possible by adiabatic capture from $56 \mathrm{MHz}$ RF into the $197 \mathrm{MHz} \mathrm{RF}$, which would maximize luminosity within vertex even further.

\begin{tabular}{|l|l|l|l|}
\hline Au@100 GeV/nucleon & $28 \mathrm{MHz}$ & $56 \mathrm{MHz}$ & $197 \mathrm{MHz}$ \\
\hline RF voltage, $\mathrm{kV}$ & 300 & 2500 & 3000 \\
\hline Bucket acceptance, eV-s/nucleon & 4.9 & 5 & 0.84 \\
\hline Bucket length, nsec & 36 & 18 & 5.1 \\
\hline initial RMS bunch length, m $\left(\mathrm{S}_{95 \%}=1.5 \mathrm{eV}-\mathrm{s} / \mathrm{n}\right)$ & & & 0.28 \\
\hline Initial full length, 5*RMS, nsec $\left(\mathrm{S}_{95 \%}=1.5 \mathrm{eV}-\mathrm{s} / \mathrm{n}\right)$ & & & 4.6 \\
\hline Initial RMS bunch length, m $\left(\mathrm{S}_{95 \%}=0.8 \mathrm{eV}-\mathrm{s} / \mathrm{n}\right)$ & 0.58 & 0.29 & 0.2 \\
\hline Initial full length, 5*RMS, nsec $\left(\mathrm{S}_{95}=0.8 \mathrm{eV}-\mathrm{s} / \mathrm{n}\right)$ & 9.7 & 4.8 & 3.4 \\
\hline
\end{tabular}

Table 1. Comparison of RF parameters for present and planned RF cavities.

For a stationary RF bucket, the bucket acceptance is give by:

$$
A_{s}=8 \frac{R}{h c} \sqrt{\frac{2 Z e V_{r f} E_{s}}{\pi h \eta}},
$$

where $R$ is the machine radius, $h$ is the RF harmonic number, $\eta$ is the slippage factor, $V_{r f}$ is the total RF gap voltage per turn, $Z$ is the ion charge number and $E_{s}$ is the energy of synchronous particles. The choice of $56 \mathrm{MHz}$ cavity with $2.5 \mathrm{MV}$ voltage results in RF bucket acceptance 6 times larger than the one of $197 \mathrm{MHz}$ RF.

IBS simulations were performed with $56 \mathrm{MHz}$ RF for ion beam parameters in Table 2 with bunch intensities of $1 \cdot 10^{9}$ and $1.5 \cdot 10^{9}$ per bunch. Simulations were performed using Martini's model of IBS in BETACOOL code [6, 7]. Presently, typical bunch intensity of Au ion beam used in RHIC operation is about $1.1 \cdot 10^{9}$. Bunch intensity of $1.5 \cdot 10^{9}$ was already used in accelerator experiments but is not yet operational due to a beam instability at the transition energy which limits average 
beam current to about $130 \mathrm{~mA}$. This corresponds to bunch intensity of $\mathrm{N}=1.3 \cdot 10^{9}$ for 103 bunches. Work in presently underway to elevate this instability threshold. Thus, operation with $1.5 \cdot 10^{9}$ (corresponds to $165 \mathrm{~mA}$ current with 112 bunches) might be possible few years from now. In Figs. 1-3 results of simulations are shown for bunch intensity of $\mathrm{N}=1.5 \cdot 10^{9}$ and $1 \cdot 10^{9}$ and longitudinal emittance of $\mathrm{S}_{95 \%}=0.8 \mathrm{eV}$-sec/nucleon (expected with $56 \mathrm{MHz} \mathrm{RF}$ ).

\begin{tabular}{|l|l|}
\hline Energy of Au ions, GeV/nucleon & 100 \\
\hline RF harmonic & 720 \\
\hline RF voltage, MV & 2.5 \\
\hline RMS bunch length, cm & 29 \\
\hline RMS momentum spread & 0.00044 \\
\hline Transverse normalized emittance,95\%, $\mu \mathrm{m}$ & 15 \\
\hline Longitudinal emittance, 95\%, eV-s/nucleon & 0.8 \\
\hline
\end{tabular}

Table 2. Initial parameters of Au ions beam used in simulations.

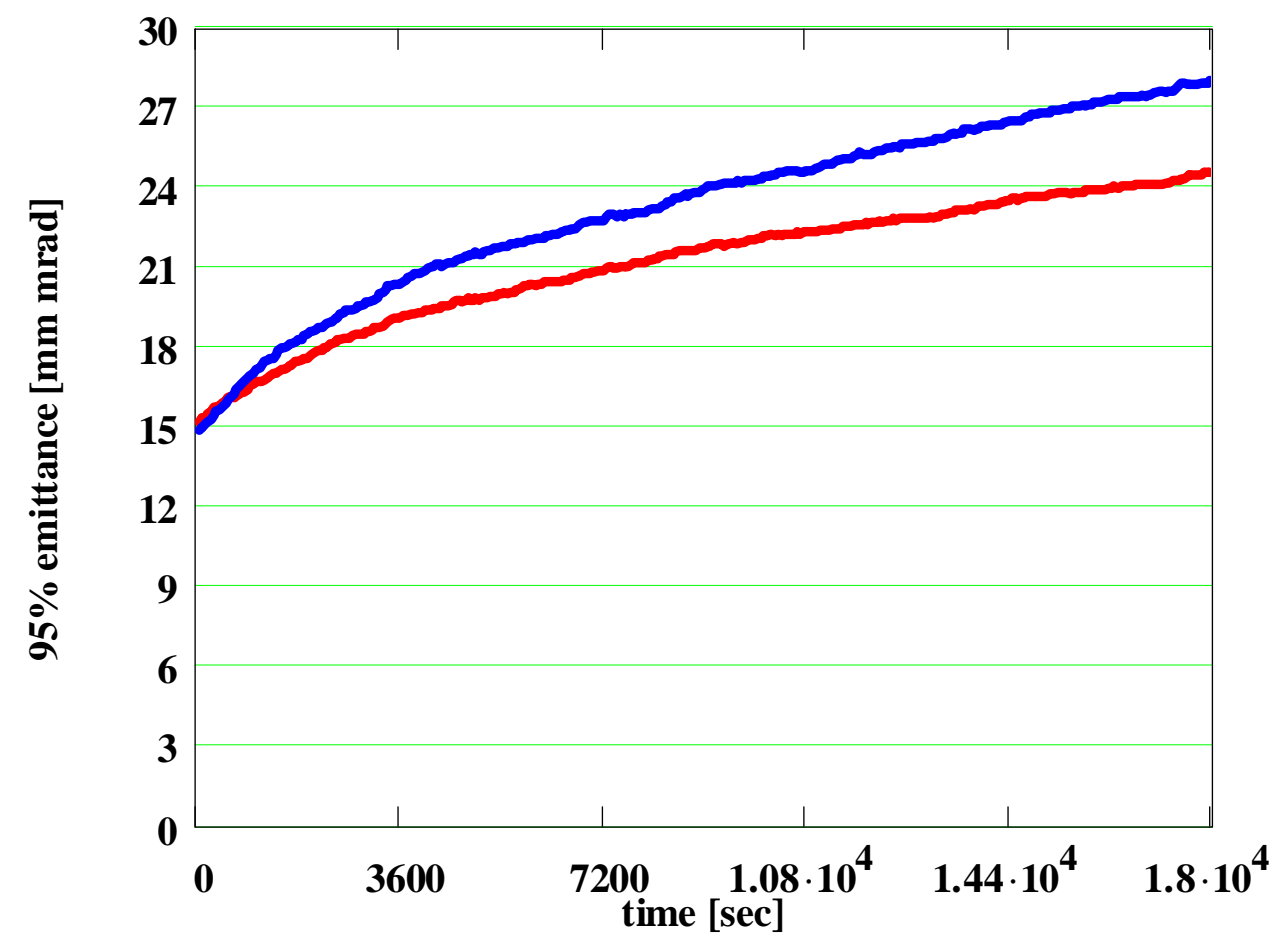

Figure 1: Evolution of 95\% normalized transverse emittance for Au ions with beam parameters in Table 2. Red lower curve - for bunch intensity $1 \times 10^{9}$; blue upper curve - for bunch intensity $1.5 \times 10^{9}$ (using IBS-lattice “dAu82” from 2008 RHIC physics run in Yellow ring). 


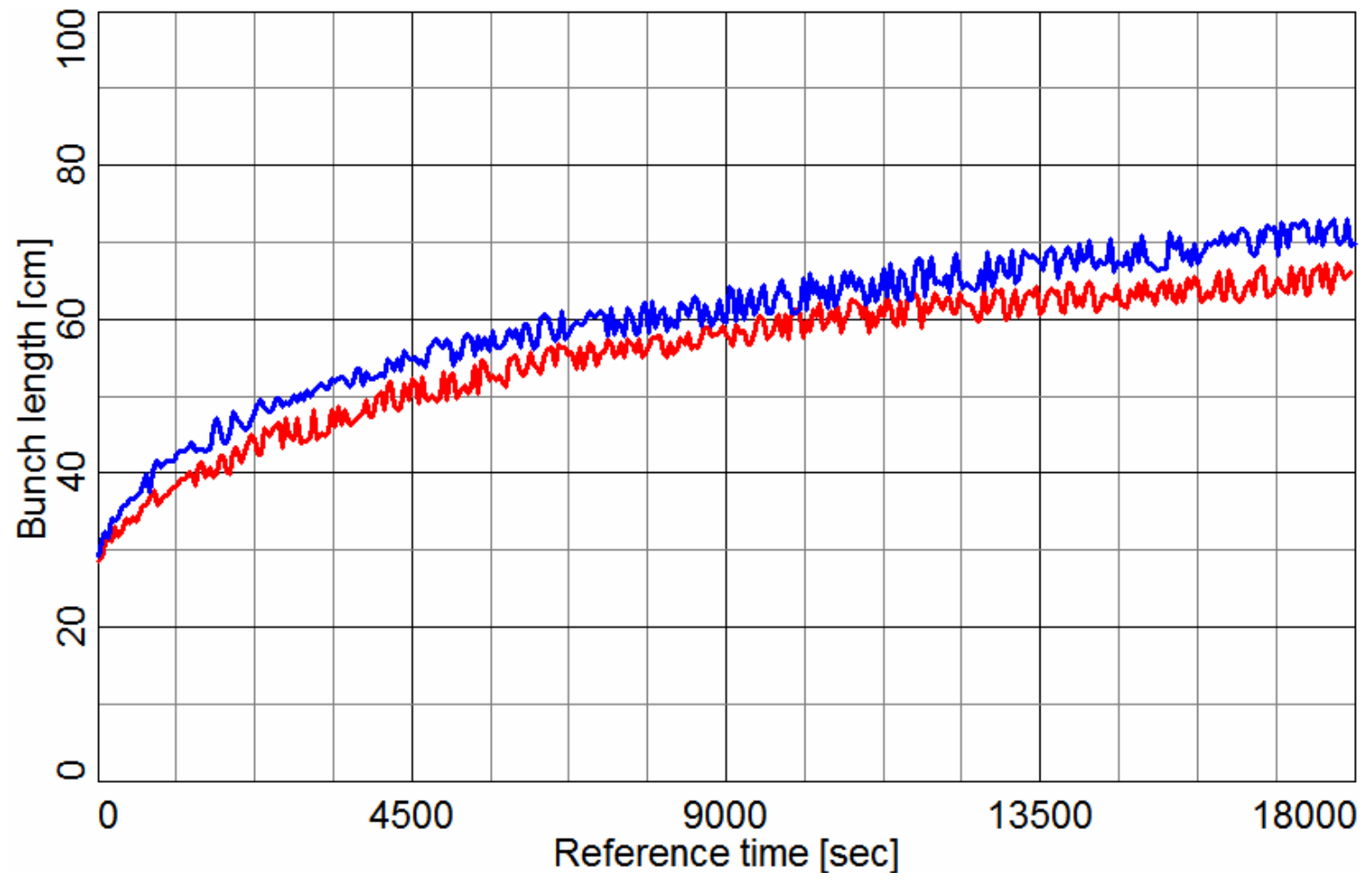

Figure 2: Evolution of RMS bunch length for Au ion bunch with beam parameters in Table 2. Red lower curve - for bunch intensity $1 \times 10^{9}$; blue upper curve - for bunch intensity $1.5 \times 10^{9}$.

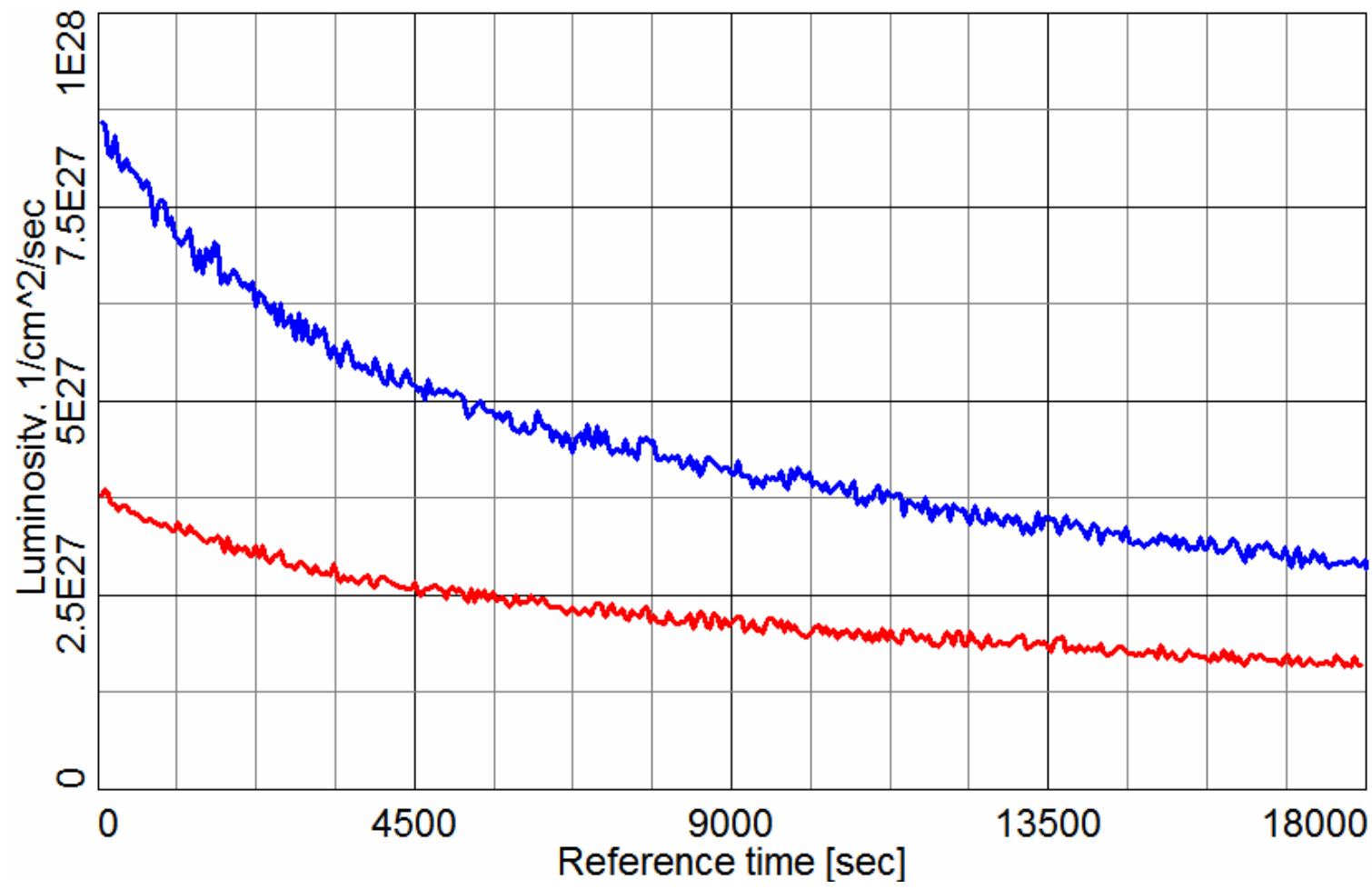

Figure 3: Resulting luminosity for $112 \mathrm{Au}$ ions bunches, $\beta^{*}=0.8 \mathrm{~m}$ for beam parameters in Table 2 . Red lower curve - for bunch intensity $1 \times 10^{9}$; blue upper curve - for bunch intensity $1.5 \times 10^{9}$. 


\begin{tabular}{|c|c|c|}
\hline Au@100 GeV/nucleon & $197 \mathrm{MHz}$ & $56 \mathrm{MHz}$ \\
\hline Run-7, $\mathrm{N}=1.1 \times 10^{9},<\mathrm{L}>_{\text {full }}, \mathrm{cm}^{-2} \mathrm{~s}^{-1}$ & $1.2 \times 10^{27}$ & \\
\hline $\mathrm{N}=1 \times 10^{9}, \beta^{*}=0.8 \mathrm{~m},<\mathrm{L}>_{\text {full }}, \mathrm{cm}^{-2} \mathrm{~s}^{-1}$ & & $2.4 \times 10^{27}$ \\
\hline $\mathrm{N}=1 \times 10^{9}, \beta^{*}=0.8 \mathrm{~m},<\mathrm{L}>_{ \pm 30 \mathrm{~cm}}, \mathrm{~cm}^{-2} \mathrm{~s}^{-1}$ & & $1 \times 10^{27}$ \\
\hline $\mathrm{N}=1.5 \times 10^{9}, \beta^{*}=0.8 \mathrm{~m},<\mathrm{L}>_{\text {full }}, \mathrm{cm}^{-2} \mathrm{~s}^{-1}$ & & $4.8 \times 10^{27}$ \\
\hline $\mathrm{N}=1.5 \times 10^{9}, \beta^{*}=0.8 \mathrm{~m},<\mathrm{L}>_{ \pm 30 \mathrm{~cm}}, \mathrm{~cm}^{-2} \mathrm{~s}^{-1}$ & & $1.9 \times 10^{27}$ \\
\hline $\mathrm{N}=1 \times 10^{9}, \beta^{*}=0.5 \mathrm{~m}$, with $3 \mathrm{D}$ stochastic cooling Ref.[8], $<\mathrm{L}>_{\text {full }}, \mathrm{cm}^{-2} \mathrm{~s}^{-1}$ & $4.3 \times 10^{27}$ & $5.5 \times 10^{27}$ \\
\hline $\mathrm{N}=1 \times 10^{9}, \beta^{*}=0.5 \mathrm{~m}$, with 3D stoch. cooling, Ref. [8], $<\mathrm{L}>_{ \pm 30 \mathrm{~cm}}, \mathrm{~cm}^{-2} \mathrm{~s}^{-1}$ & $3 \times 10^{27}$ & $4 \times 10^{27}$ \\
\hline
\end{tabular}

Table 3. Expected luminosity performance with future $56 \mathrm{MHz}$ RF upgrade. Subscript "full” indicates full luminosity without the vertex cut, while subscript “ $\pm 30 \mathrm{~cm}$ ” corresponds to luminosity within the vertex cut of $\pm 30 \mathrm{~cm}$. Luminosity values for the last two rows which show performance with addition of a full 3D stochastic cooling upgrade were taken from simulations done by $\mathrm{M}$. Blaskiewicz [8, 9].

To summarize, the $56 \mathrm{MHz}$ SRF cavity upgrade offers 6 times larger bucket acceptance compared to the $197 \mathrm{MHz} \mathrm{RF}$ and shorter bunch lengths compared to the $28 \mathrm{MHz} \mathrm{RF}$. It also eliminates intensity spill in the neighbouring buckets and avoids longitudinal emittance increase, which happens during re-bucketing. Comparison of different RF systems is summarized in Table 1. Note that for small longitudinal emittances of $0.5-0.8 \mathrm{eV}-\mathrm{s} /$ nucleon (which is expected at top energy without re-bucketing), the $56 \mathrm{MHz}$ RF provides bunches less than $5 \mathrm{nsec}$ long which allows further reduction of bunch length with adiabatic capture into $197 \mathrm{MHz}$ RF without the need of re-bucketing.

Expected improvement in average beam luminosity is summarized for different bunch intensities in Table 3. For bunch intensity $\mathrm{N}=1.5 \cdot 10^{9}$, without stochastic cooling, one gets full luminosity without vertex cut $<\mathrm{L}>_{\text {full }}=4.8 \cdot 10^{27} \mathrm{~cm}^{-2} \mathrm{~s}^{-1}$ in a 4-hour store (for 112 bunches and $\beta^{*}=0.8$ meters), and $<\mathrm{L}>_{\text {full }}=2.4 \cdot 10^{27} \mathrm{~cm}^{-2} \mathrm{~s}^{-1}$ for $\mathrm{N}=1.0 \cdot 10^{9}$. The best luminosity performance is expected with both $56 \mathrm{MHz}$ RF cavity and all-plane (3D) stochastic cooling upgrades which should provide around $<\mathrm{L}>_{\text {full }}=5.5 \cdot 10^{27} \mathrm{~cm}^{-2} \mathrm{~s}^{-1}$ (with $\beta^{*}=0.5$ meters) for bunch intensity of $\mathrm{N}=1.0 \bullet 10^{9}$ [8] and up to $<\mathrm{L}>_{\text {full }}=7 \cdot 10^{27} \mathrm{~cm}^{-2} \mathrm{~s}^{-1}$ for bunch intensity of $\mathrm{N}=1.5 \cdot 10^{9}[9]$.

\section{Performance of proton beams at top energy of $250 \mathrm{GeV}$}

This section summarises expected performance for protons beams with $59 \mathrm{MHz}$ cavity upgrade. Comparison of RF parameters for present $28 \mathrm{MHz}$ and $197 \mathrm{MHz}$ RF and planned $56 \mathrm{MHz}$ $\mathrm{RF}$ is given in Table 4. The choice of $56 \mathrm{MHz}$ cavity with $2.5 \mathrm{MV}$ voltage results in RF bucket acceptance 6 times larger than the one of $197 \mathrm{MHz}$.

\begin{tabular}{|l|l|l|l|}
\hline p @ 250 GeV & $28 \mathrm{MHz}$ & $56 \mathrm{MHz}$ & $197 \mathrm{MHz}$ \\
\hline Harmonic number & 360 & 720 & 2520 \\
\hline RF voltage, kV & 300 & 2500 & 3000 \\
\hline Bucket length, nsec & 35.5 & 17.8 & 5.1 \\
\hline Bucket acceptance, eV-s & 13.9 & 14.2 & 2.4 \\
\hline
\end{tabular}

Table 4. Comparison of RF parameters for present and planned RF cavities.

Up to now, operation with protons was limited by large longitudinal emittance at the top energy of $100 \mathrm{GeV}$. The emittance was intentionally increased by mismatch at injection to avoid transverse emittance growth. With the ongoing $9 \mathrm{MHz}$ cavity upgrade operation with smaller longitudinal 
emittance (0.5-1 eV-s) may become possible. The goal of $9 \mathrm{MHz}$ cavity is to get long bunches with low longitudinal emittance on the ramp to prevent transverse emittance growth suspected to be driven by electron cloud [10]. At the top energy of $250 \mathrm{GeV}$ such small longitudinal emittance would result in smaller bunch length with $28 \mathrm{MHz}$ RF. The $56 \mathrm{MHz}$ RF cavity offers even smaller bunch length thus improving vertex luminosity compared to operation with the $28 \mathrm{MHz} \mathrm{RF}$, for any value of the longitudinal emittance. In addition, for small longitudinal emittances the $56 \mathrm{MHz} \mathrm{RF}$ will allow even further shortening of the length of proton bunch with an adiabatic capture in $197 \mathrm{MHz}$ RF without a need of re-bucketing. Tables 5-7 shows expected bunch lengths for different longitudinal beam emittances.

\begin{tabular}{|l|l|l|l|}
\hline $\mathrm{p} @ 250 \mathrm{GeV}, \mathrm{S}=2 \mathrm{eV}-\mathrm{s}$ & $28 \mathrm{MHz}$ & $56 \mathrm{MHz}$ & $197 \mathrm{MHz}$ \\
\hline RF voltage, $\mathrm{kV}$ & 300 & 2500 & 3000 \\
\hline RMS bunch length, meters & 0.6 & 0.3 & 0.2 \\
\hline RMS bunch length, nsec & 2 & 1 & 0.7 \\
\hline Full bunch length (5•RMS), nsec & 10 & 5 & 3.5 \\
\hline
\end{tabular}

Table 5. Expected initial bunch length for present longitudinal emittance of $2 \mathrm{eV}$-s.

\begin{tabular}{|l|l|l|l|}
\hline $\mathrm{p}$ @ 250 GeV, S=1 eV-s & $28 \mathrm{MHz}$ & $56 \mathrm{MHz}$ & $197 \mathrm{MHz}$ \\
\hline RF voltage, $\mathrm{kV}$ & 300 & 2500 & 3000 \\
\hline RMS bunch length, meters & 0.42 & 0.21 & 0.15 \\
\hline RMS bunch length, nsec & 1.4 & 0.7 & 0.5 \\
\hline Full bunch length (5•RMS), nsec & 7 & 3.5 & 2.5 \\
\hline
\end{tabular}

Table 6. Expected initial bunch length for longitudinal emittance of $1 \mathrm{eV}$-s.

\begin{tabular}{|l|l|l|l|}
\hline $\mathrm{p}$ @ 250 GeV, S=0.5 eV-s & $28 \mathrm{MHz}$ & $56 \mathrm{MHz}$ & $197 \mathrm{MHz}$ \\
\hline RF voltage, $\mathrm{kV}$ & 300 & 2500 & 3000 \\
\hline RMS bunch length, meters & 0.3 & 0.15 & 0.1 \\
\hline RMS bunch length, nsec & 1 & 0.5 & 0.3 \\
\hline Full bunch length (5•RMS), nsec & 5 & 2.5 & 1.5 \\
\hline
\end{tabular}

Table 7. Expected initial bunch length for longitudinal emittance of $0.5 \mathrm{eV}$-s.

Below we show BETACOOL simulation of beam evolution due to IBS first with present longitudinal emittance of $2 \mathrm{eV}$-s and then with smaller emittance of $1 \mathrm{eV}$-s (assuming operation with smaller emittance will be possible in the future). Simulations assumed separate operation either with $56 \mathrm{MHz}$ or $197 \mathrm{MHz} \mathrm{RF}$.

\begin{tabular}{|l|l|l|}
\hline Protons @ 250 GeV & $56 \mathrm{MHz}$ cavity & $197 \mathrm{MHz}$ cavity \\
\hline RF bucket acceptance, eV-s & 14.2 & 2.4 \\
\hline Bunch intensity & $2 \times 10^{11}$ & $2 \times 10^{11}$ \\
\hline RF voltage, MV & 2.5 & 3.0 \\
\hline RMS bunch length, cm & 32 & 22 \\
\hline RMS momentum spread & 0.00041 & 0.00058 \\
\hline Longitudinal emittance, 95\%, eV-s & 2.0 & 2.0 \\
\hline Transverse normalized emittance, 95\%, $\mu \mathrm{m}$ & 15 & 15 \\
\hline
\end{tabular}

Table 8. Initial parameters of proton beam with $2 \mathrm{eV}$-s longitudinal emittance used in simulations. 


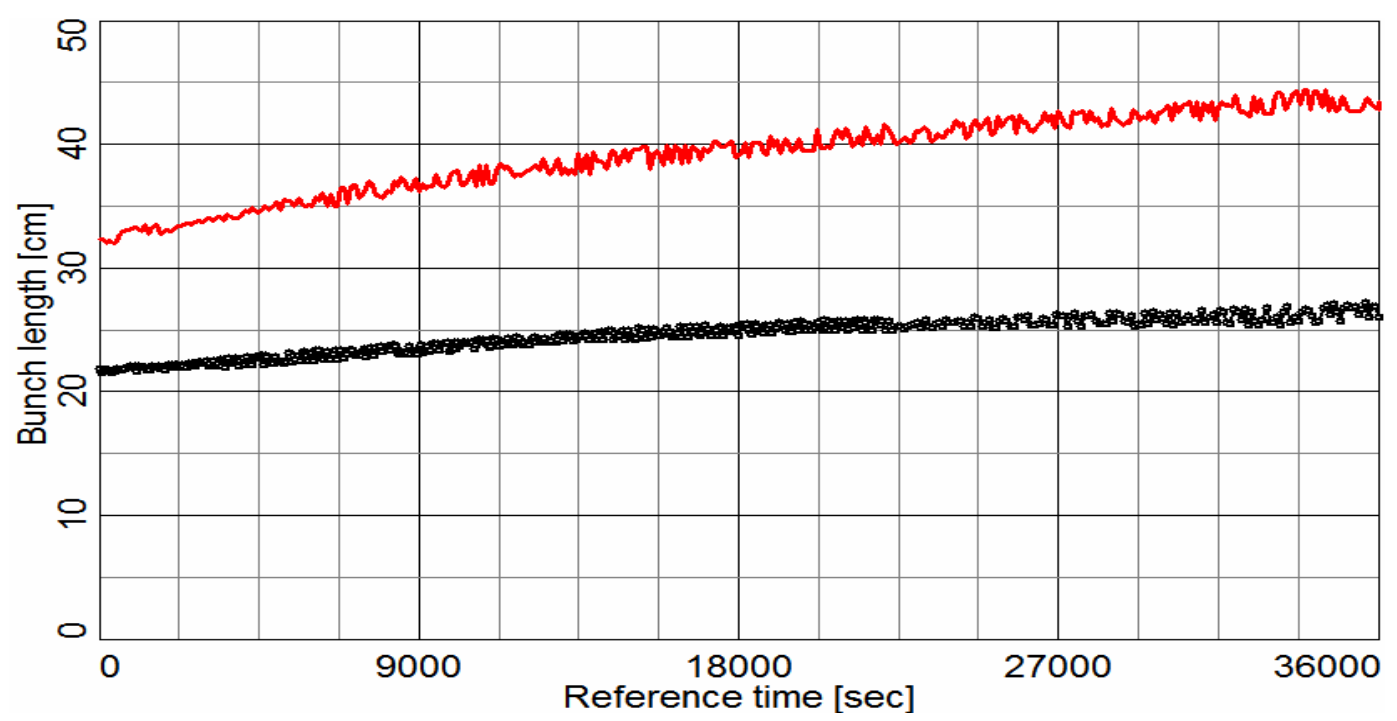

Figure 7: Evolution of RMS bunch length due to IBS for beam parameters in Table 8. Top red curve: for 56MHz RF; lower black curve for 197MHz RF.

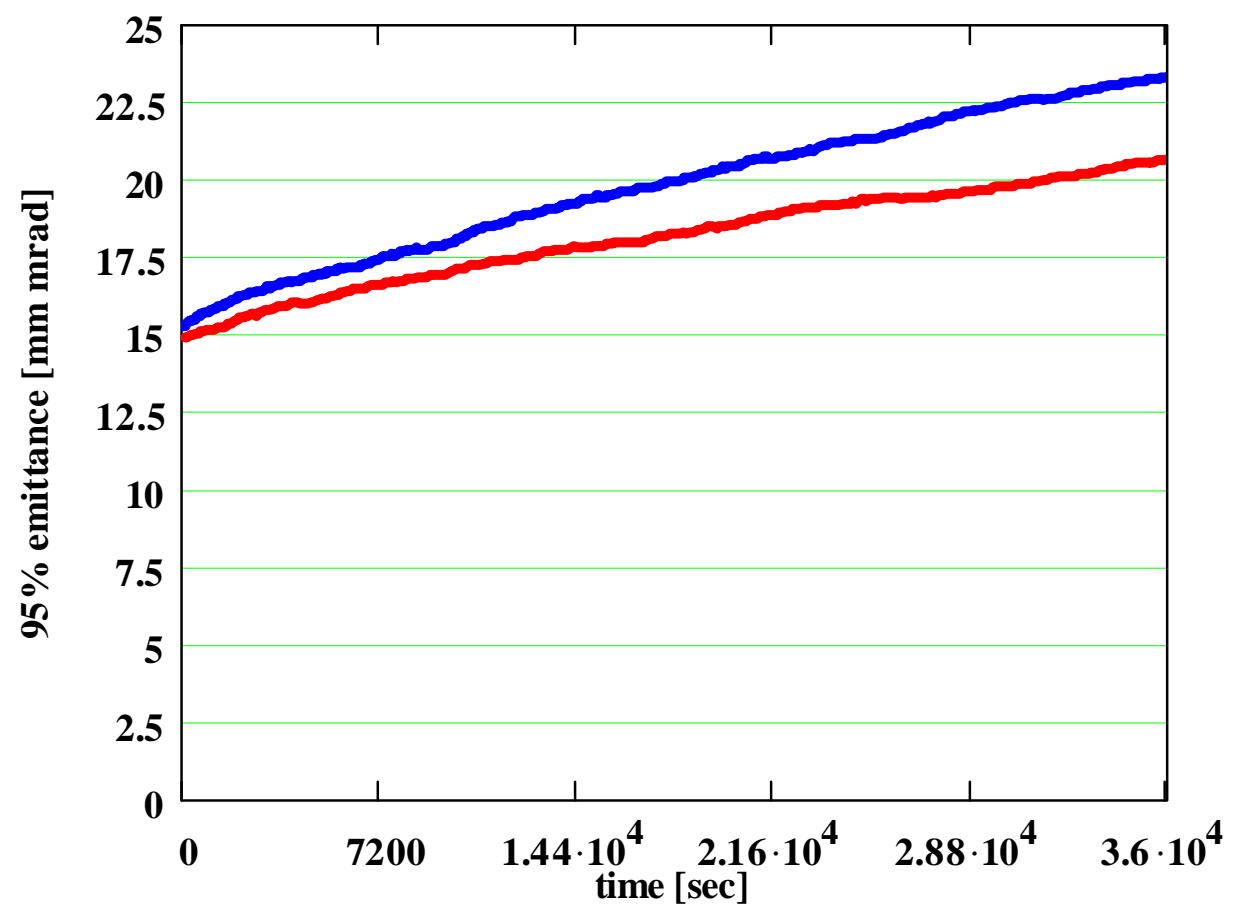

Figure 8. Evolution of 95\% normalized transverse beam emittance due to IBS for beam parameters in Table 8. Lower red curve: for 56MHz RF; upper blue curve for $197 \mathrm{MHz} \mathrm{RF}$. 


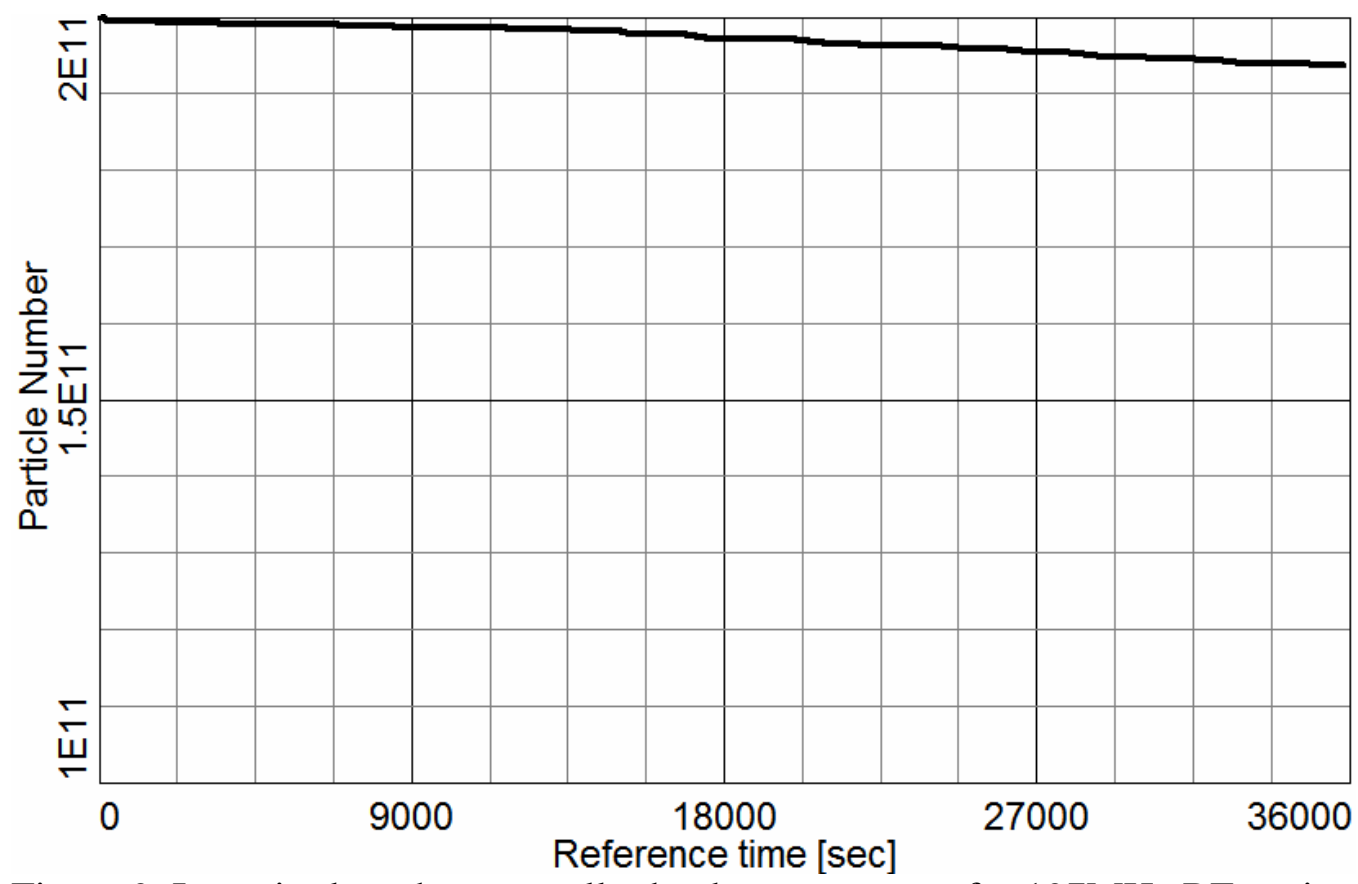

Figure 9. Intensity loss due to smaller bucket acceptance for 197MHz RF cavity.

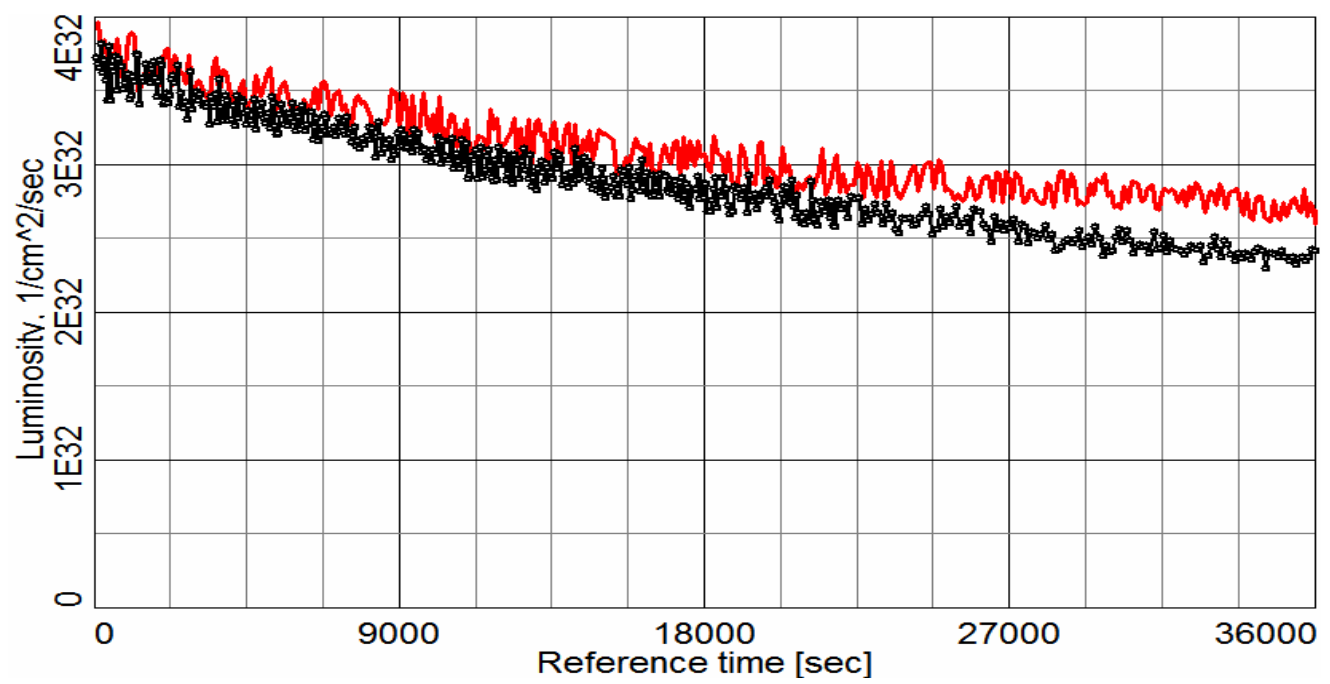

Figure 10. Full luminosity (without vertex cut) with 112 bunches, $\beta^{*}=0.8 \mathrm{~m}$ for $56 \mathrm{MHz}$ (red curve) and 197MHz RF (black curve).

Figures 11-14 show beam evolution due to IBS for the case of smaller initial longitudinal emittance of $1 \mathrm{eV}$-sec and beam parameters shown in Table 9.

\begin{tabular}{|l|l|l|}
\hline Protons @ 250 GeV & $56 \mathrm{MHz}$ cavity & $197 \mathrm{MHz}$ cavity \\
\hline RF bucket acceptance. eV-s & 14.2 & 2.4 \\
\hline Bunch intensity & $2 \times 10^{11}$ & $2 \times 10^{11}$ \\
\hline RF voltage, MV & 2.5 & 3.0 \\
\hline RMS bunch length, cm & 22 & 16 \\
\hline RMS momentum spread & 0.00029 & 0.00042 \\
\hline Longitudinal emittance, 95\%, eV-s & 1.0 & 1.0 \\
\hline Transverse normalized emittance, 95\%, $\mu \mathrm{m}$ & 15 & 15 \\
\hline
\end{tabular}

Table 9. Initial parameters of proton beam with $1 \mathrm{eV}$-s longitudinal emittance used in simulations. 


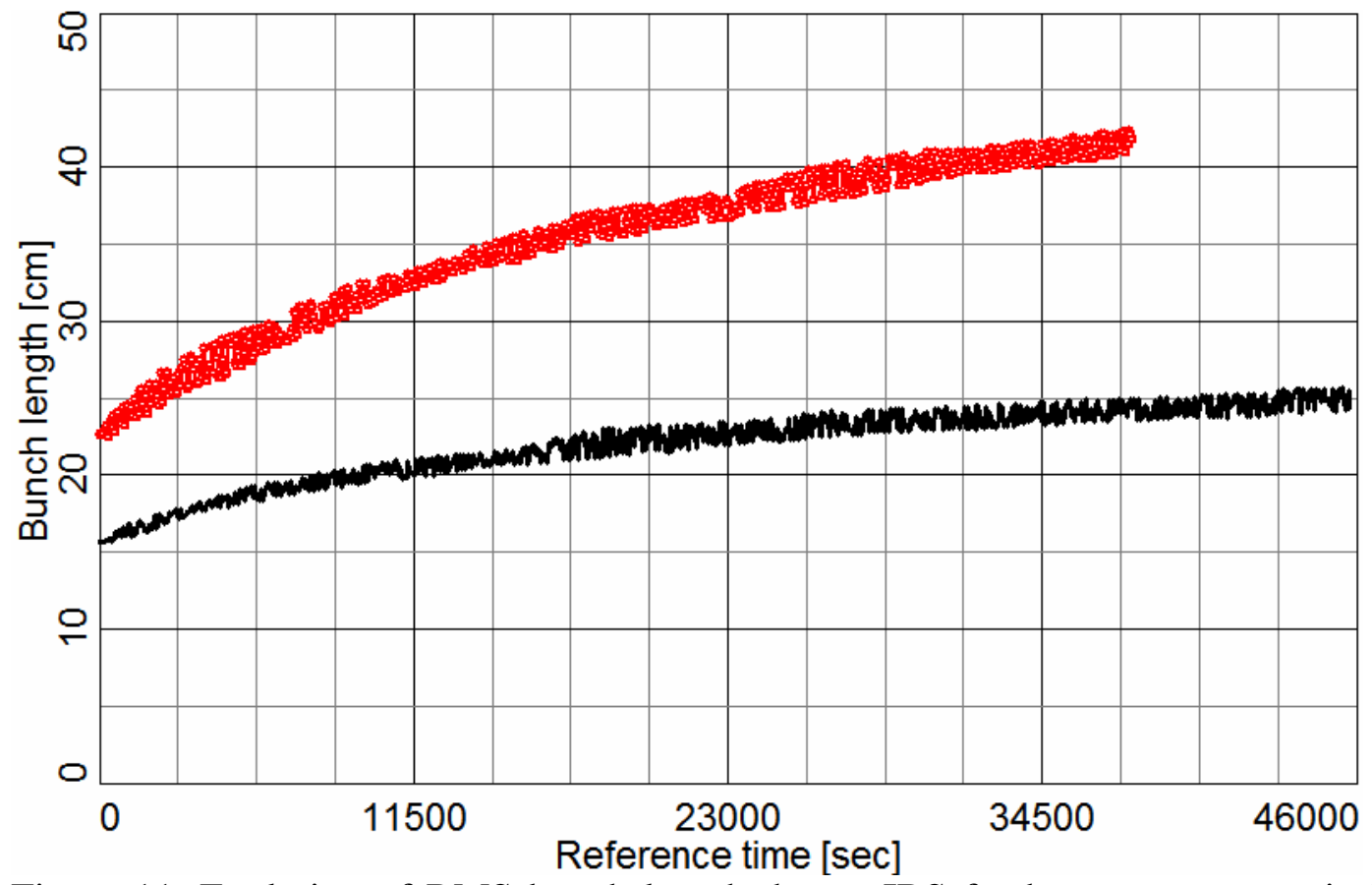

Figure 11. Evolution of RMS bunch length due to IBS for beam parameters in Table 9. Top red curve: for 56MHz RF; lower black curve for 197MHz RF.

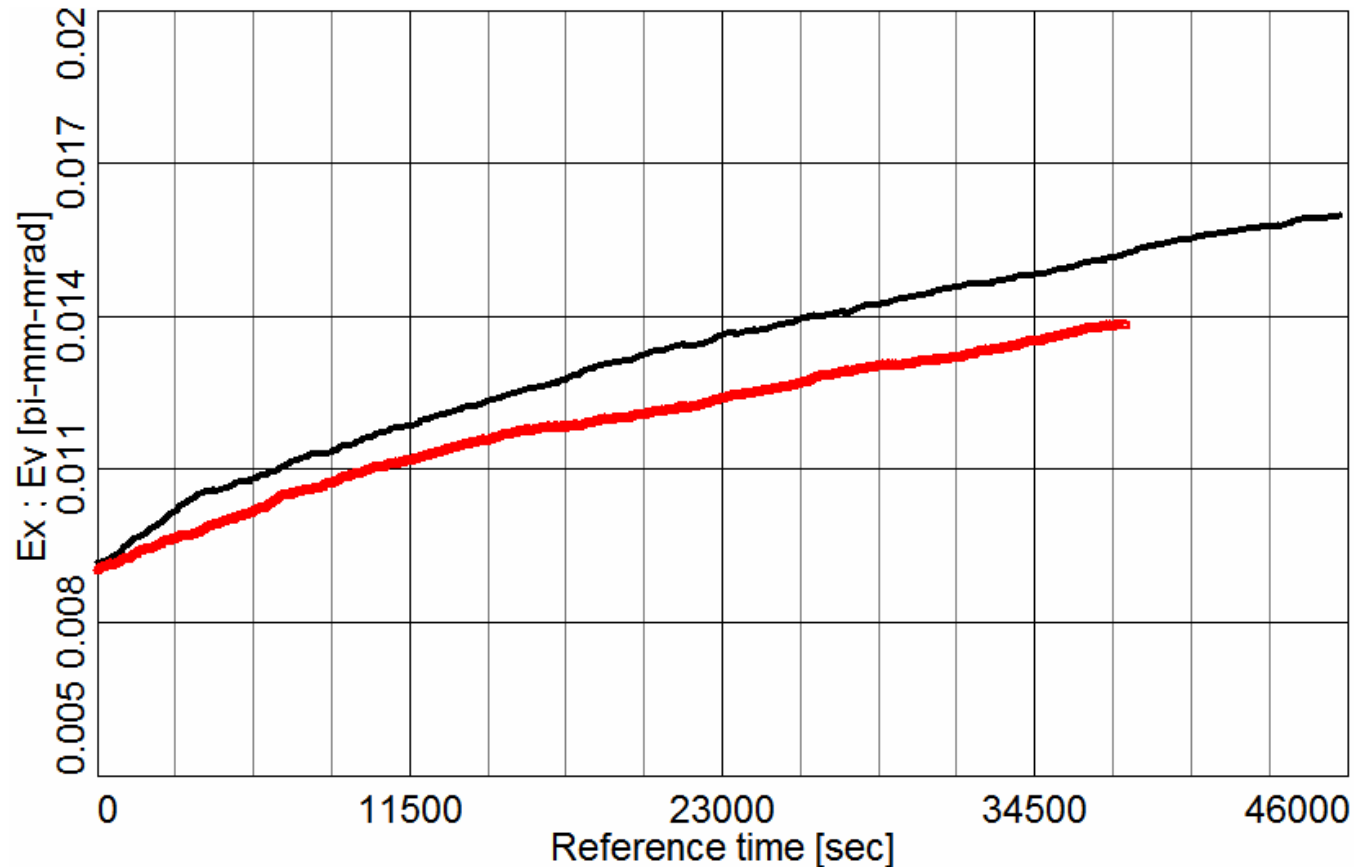

Figure 12: Evolution of RMS un-normalized transverse beam emittance due to IBS for beam parameters in Table 9. Lower red curve: for 56MHz RF; upper black curve for 197MHz RF. 


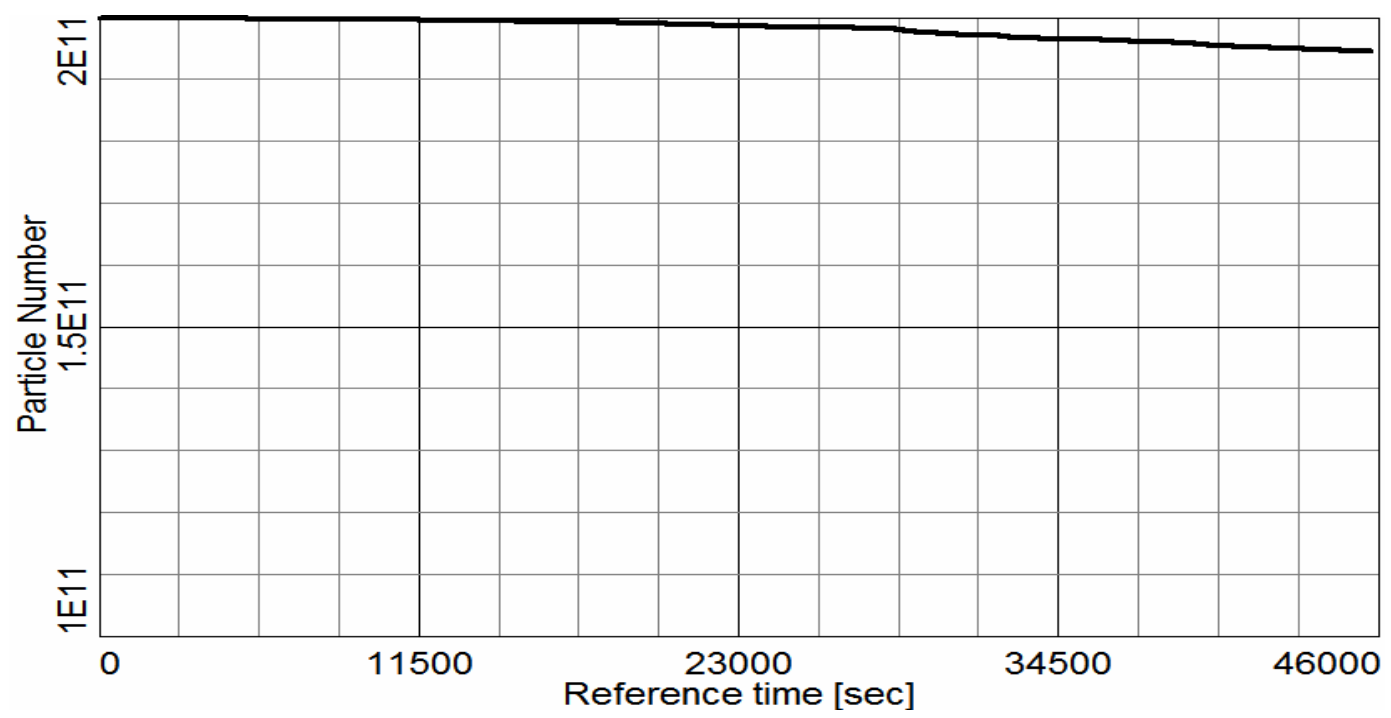

Figure 13: Intensity loss due to smaller bucket acceptance for 197MHz RF cavity.

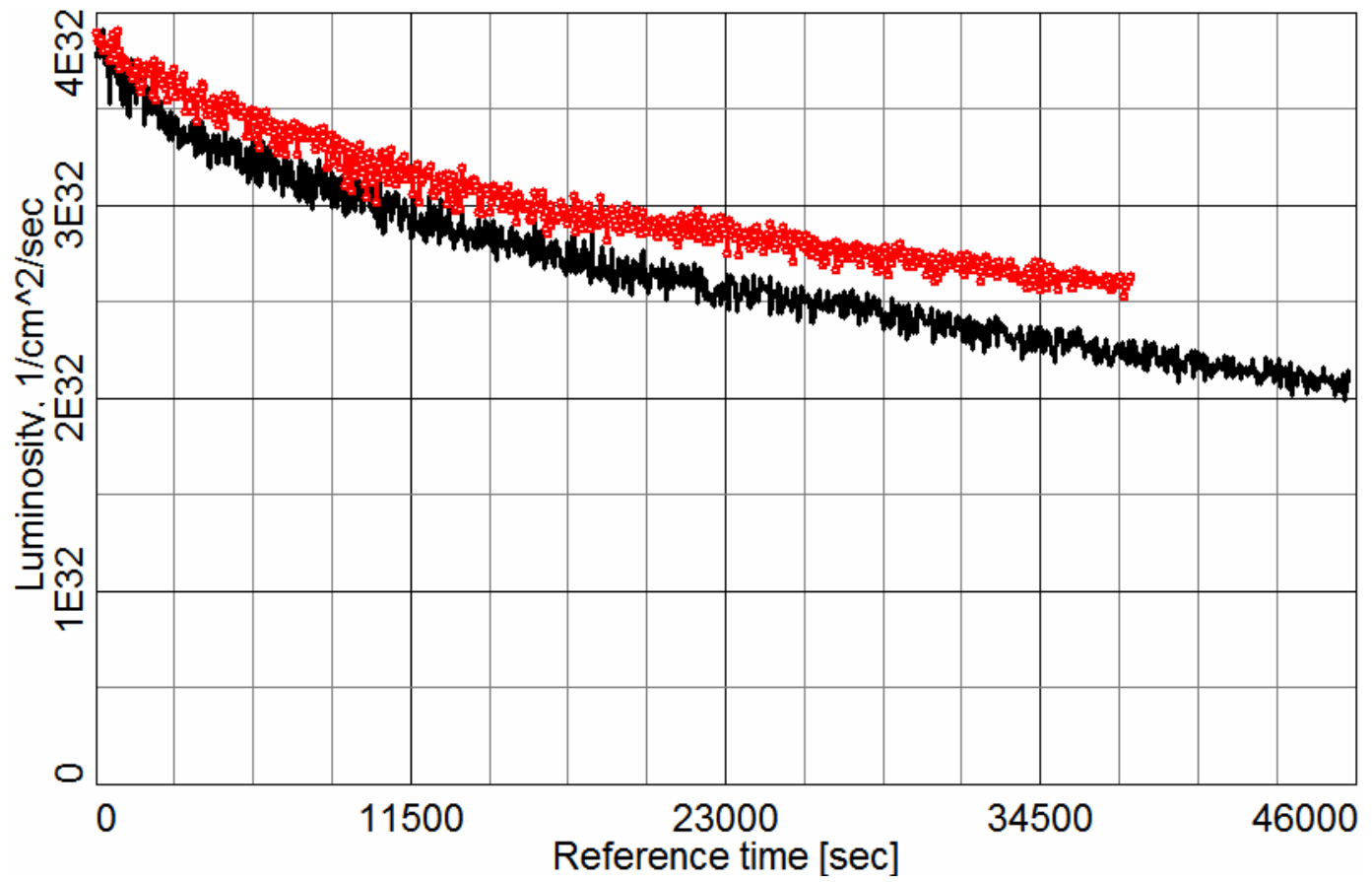

Figure 14. Full luminosity (without vertex cut) with 112 bunches, $\beta^{*}=0.8 \mathrm{~m}$ for $56 \mathrm{MHz}$ (red curve) and 197MHz RF (black curve).

To summarize, for protons at $250 \mathrm{GeV}$, planned $56 \mathrm{MHz}$ SRF cavity upgrade offers significantly better vertex luminosity compared to present $28 \mathrm{MHz}$ RF due to shorter bunch length for any value of the longitudinal emittance (see Tables 5-7). Luminosity performance with $56 \mathrm{MHz}$ RF seems to be slightly better than with $197 \mathrm{MHz}$ cavity for beam parameters shown in Tables 8-9. Compared to $197 \mathrm{MHz}$ no intensity loss due to longitudinal IBS is expected because of significantly larger bucket acceptance for 56MHz SRF cavity. In addition, the $56 \mathrm{MHz}$ RF offers possibility of further shortening of proton bunches with adiabatic capture into $197 \mathrm{MHz} \mathrm{RF}$. 


\section{Possible improvement of Au ion luminosity with electron cooling at top energy}

Electron cooling of Au ions in RHIC at $100 \mathrm{GeV} /$ nucleon was carefully studied for RHIC-II project [2]. The most recent version of the cooler used simplified parameters without a need to move triplets in RHIC [11]. It is expected that RHIC-II average luminosities of $<\mathrm{L}>=5-7 \cdot 10^{27} \mathrm{~cm}^{-2} \mathrm{sec}^{-1}$ can be reached with a full 3D stochastic cooling system and $56 \mathrm{MHz}$ SRF cavity upgrades. Presently there is no plan to have an additional high-energy electron cooling system for Au ions at $100 \mathrm{GeV} /$ nucleon. For completeness, in this section we show potential improvement in luminosity if one would have such high-energy electron cooling system as well.

\begin{tabular}{|l|l|}
\hline Kinetic energy, $\mathrm{MeV}$ & 54 \\
\hline Total length of 2 cooling section, $\mathrm{m}$ & 50 \\
\hline Charge per bunch, $\mathrm{nC}$ & 5.0 \\
\hline Total charge in bunch train (3 bunches), nC & 15 \\
\hline RMS momentum spread & 0.0005 \\
\hline RMS normalized emittance, $\mu \mathrm{m}$ & 3 \\
\hline Undulator: period, magnetic field & $8 \mathrm{~cm}, 10 \mathrm{G}$ \\
\hline
\end{tabular}

Table 10. Parameters of electron cooler used for simulations in Fig. 15.

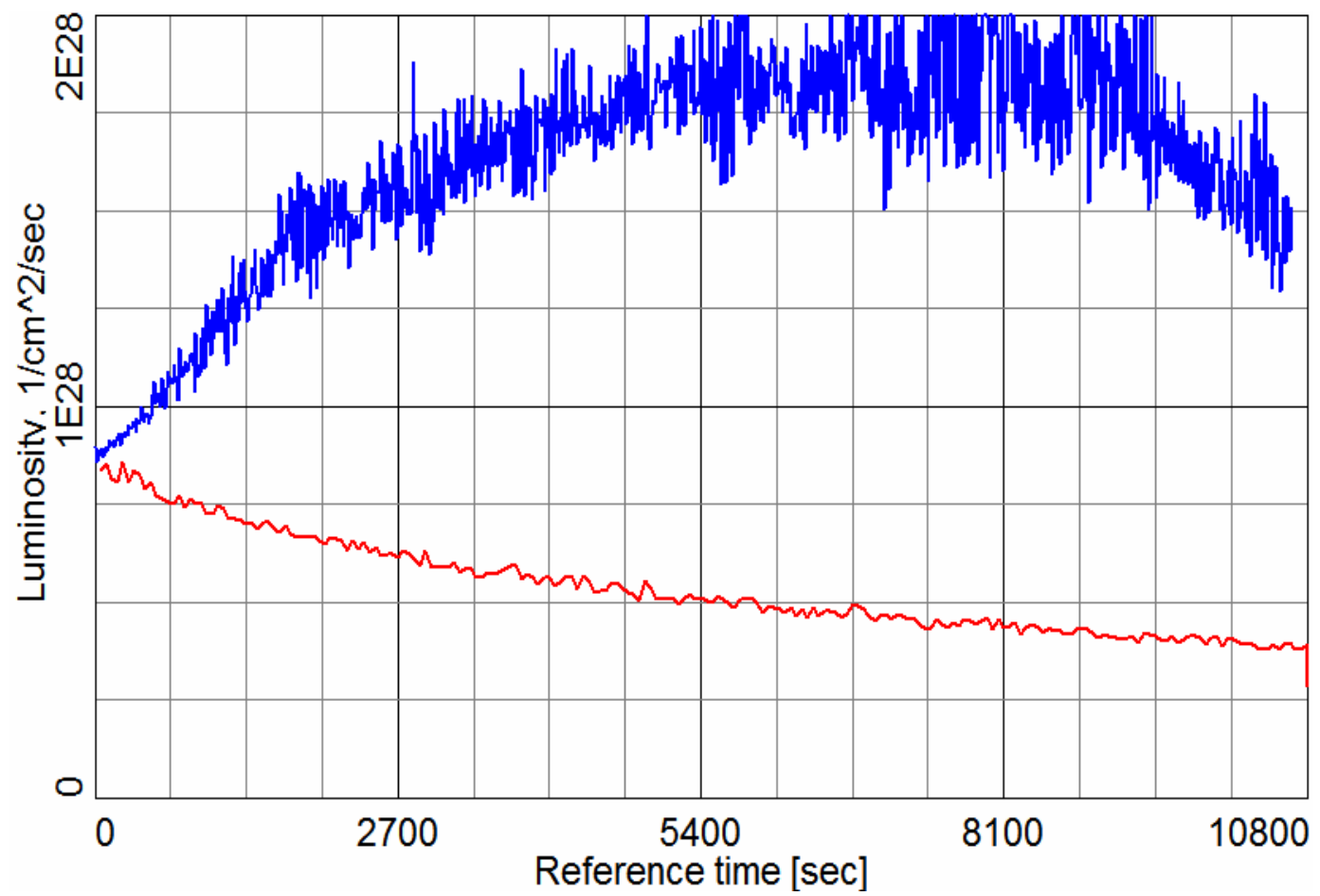

Figure 15. Luminosity of $\mathrm{Au}$ ions at $100 \mathrm{GeV} /$ nucleon for ion bunch intensity of $\mathrm{N}_{\mathrm{i}}=1.5 \cdot 10^{9}$, $\beta^{*}=0.8 \mathrm{~m}$ : with electron cooling (blue upper curve) and without cooling (red lower curve).

Without electron cooling average luminosity in 3-hour store is $<\mathrm{L}>=5.4 \cdot 10^{27} \mathrm{~cm}^{-2} \mathrm{sec}^{-1}$ without vertex cut and $<\mathrm{L}>=2.7 \cdot 10^{27} \mathrm{~cm}^{-2} \mathrm{sec}^{-1}$ with the vertex cut of $\pm 30 \mathrm{~cm}$ (without stochastic cooling). With electron cooling expected average luminosity in 3-hour store shown in Fig. 15 is $<\mathrm{L}>=1.6 \bullet 10^{28}$ $\mathrm{cm}^{-2} \mathrm{sec}^{-1}$ (with $\mathrm{N}_{\mathrm{i}}=1.5 \cdot 10^{9}$ ). Since electron cooling also keeps short bunch length within the vertex cut it would provide close to a six-fold improvement in luminosity within the vertex compared to operation with $56 \mathrm{MHz}$ SRF cavity along, and about two-fold improvement compared to the 
expected future performance with full 3D stochastic cooling system and $56 \mathrm{MHz} \mathrm{RF}$ upgrades combined [9].

\section{Acknowledgement}

We would like to thank Ilan Ben-Zvi, Mike Blaskiewicz, Mike Brennan and Wolfram Fischer for useful discussions on this subject. In simulations, we used BETACOOL code developed at JINR, Dubna, Russia.

\section{References}

[1] RHIC design Manual, 1998.

[2] http://www.bnl.gov/cad/ecooling

[3] M. Blaskiewicz et al., Proc. of PAC07 (Albuquerque, NM, USA), p. 2014.

[4] V. Litvinenko et al., Proc. of EPAC08 (Genoa, Italy), p. 2557 (2008).

[5] A. Fedotov et al., Proc. of HB2008 workshop (Nashville, TN, August 2008).

[6] BETACOOL code, http://lepta.jinr.ru; A. Sidorin et al., NIM A 558, p. 325 (2006).

[7] Physics Guide of BETACOOL code, BNL Tech Note C-A/AP/\#262 (2006).

[8] M. Blaskiewicz, private communication (2008).

[9] M. Blaskiewicz, presentation at RHIC MAC08 (BNL, 2008).

[10] S.Y. Zhang and V. Ptitsyn, Phys. Rev. STAB, 11, 051001 (2008).

[11] A. Fedotov (October 2007), unpublished. 